> La perte des mécanismes de contrôle de la progression du cycle de division cellulaire ou du déclenchement des processus d'apoptose permet aux cellules d'acquérir des propriétés décisives pour leur transformation tumorale. Plusieurs protéine kinases participent à la transduction de signaux qui neutralisent des composants de la machinerie apoptotique. Dans ce contexte, la protéine kinase CK2 (caséine kinase 2), dont la structure vient d'être élucidée, apparaît comme un régulateur déterminant pour la viabilité cellulaire. II est concevable que la surexpression de la CK2 observée dans les cancers puisse conduire à la formation de signaux de survie contribuant à la tumorigenèse. <

\section{La protéine kinase CK2, \\ une enzyme qui cultive la différence}

Thierry Buchou, Claude Cochet

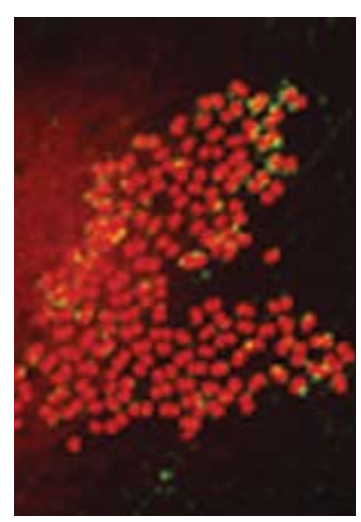

vation au cours de l'évolution, sont des propriétés indicatives d'une fonction critique de cette enzyme dans la cellule. Il faut cependant admettre que, malgré d'intenses efforts, la place de la CK2 dans la vie de la cellule est demeurée, jusqu'à ce jour, mystérieuse. Notamment, aucun second messager réglant son activité ou sa localisation n'a été identifié à ce jour. Néanmoins, des observations récentes suscitent un regain d'intérêt pour cette protéine kinase. Cet article résume les travaux ayant permis d'élucider son étonnante architecture moléculaire et de visualiser la localisation dynamique de ses sous-unités dans la cellule, et tente de proposer un mode de régulation. La connaissance des mécanismes mis en jeu dans cette régulation est capitale pour établir la place de cette protéine kinase dans le contrôle de la prolifération, de la survie et de la transformation cellulaire.

\section{La CK2 à l'échelle atomique}

Comparée aux autres protéine kinases, la CK2 possède plusieurs caractéristiques non conventionnelles sur le plan de son organisation moléculaire. La complexité de 
cette protéine kinase est déjà évidente au niveau de sa structure quaternaire. Purifiée à partir de différentes sources, l'enzyme se présente sous la forme d'un complexe moléculaire tétramérique (holoenzyme) composé de deux sous-unités catalytiques $($ CK2 $\alpha)$ et de deux sous-unités régulatrices (CK2 $\beta$ ). Deux gènes codant pour les deux isoformes de la sousunité catalytique (CK2 $\alpha$ et CK2 $\alpha^{\prime}$ ) sont présents dans le génome de la plupart des organismes. L'holoenzyme peut donc se retrouver sous la forme de complexes de type $\alpha_{2} \beta_{2}, \alpha \alpha^{\prime} \beta_{2}, \alpha_{2}^{\prime} \beta_{2}$ [1].

La structure tertiaire de chaque sous-unité a été résolue (Figure 1). Comme toutes les protéine kinases, la sous-unité catalytique présente une structure bilobée composée d'un domaine aminoterminal riche en feuillets $\beta$ et d'un domaine carboxy-terminal riche en hélices $\alpha$. Les deux lobes délimitent le site catalytique qui, dans le cas de la CK2, est largement accessible en raison d'une forte interaction entre l'extrémité amino-terminale de la kinase et sa boucle d'activation, segment de la protéine contrôlant l'accès des substrats à la poche catalytique [2].

La conformation particulère de son site catalytique explique que CK2 soit l'une des rares protéine kinases utilisant indifféremment I'ATP ou le GTP comme nucléotide donneur de phosphate. Elle rend également compte du fait que, contrairement à la plupart des protéine kinases, la sous-unité catalytique de CK2 est constitutivement active, qu'elle soit ou non associée à la sous-unité $\beta$. Cette activité basale est néanmoins fortement modulée par la présence de CK2 $\beta$. Ainsi, la phosphorylation de nombreux substrats de l'enzyme dépend strictement de la présence de la sous-unité $\beta$, qui possède donc les propriétés d'une authentique sous-unité régulatrice. Face à l'étonnante diversité de ses substrats (plus de 200 identifiés in vitro), la présence ou l'absence de la sous-unité régulatrice permet un ciblage des substrats qui peuvent alors se classer en trois familles (Figure 2).

À l'examen de sa séquence primaire, CK2 $\beta$ ne présente d'homologie avec aucune protéine connue. Sa structure (FigurelB) révèle l'architecture d'un dimère dont l'interface, extrêmement réduite, repose sur des interac-

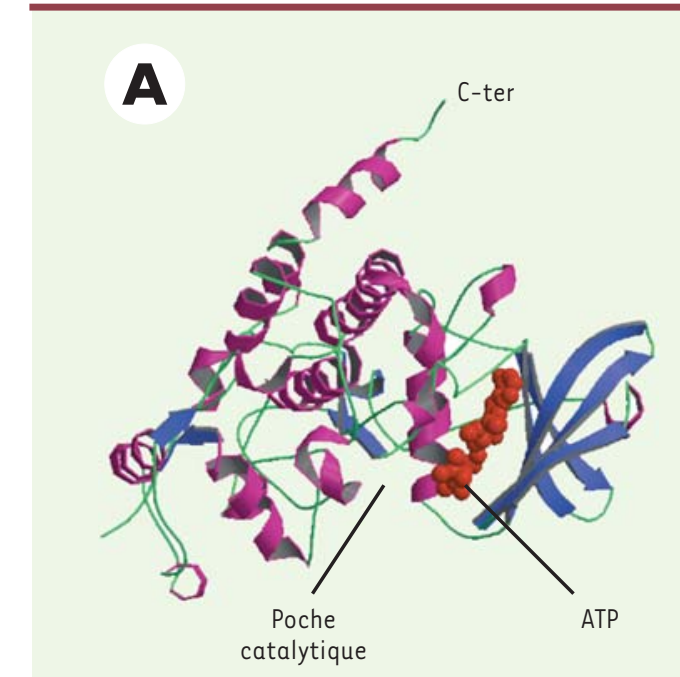

B

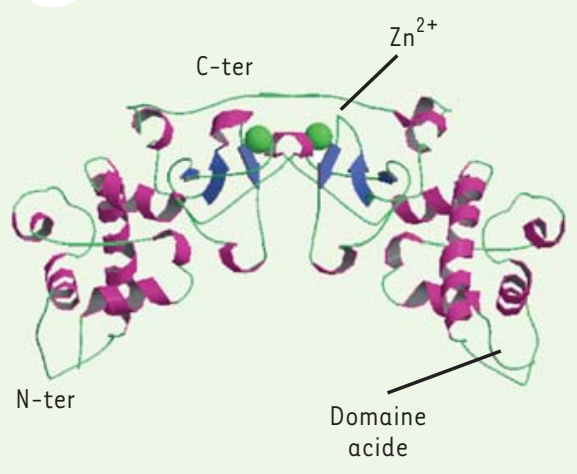
catalytique

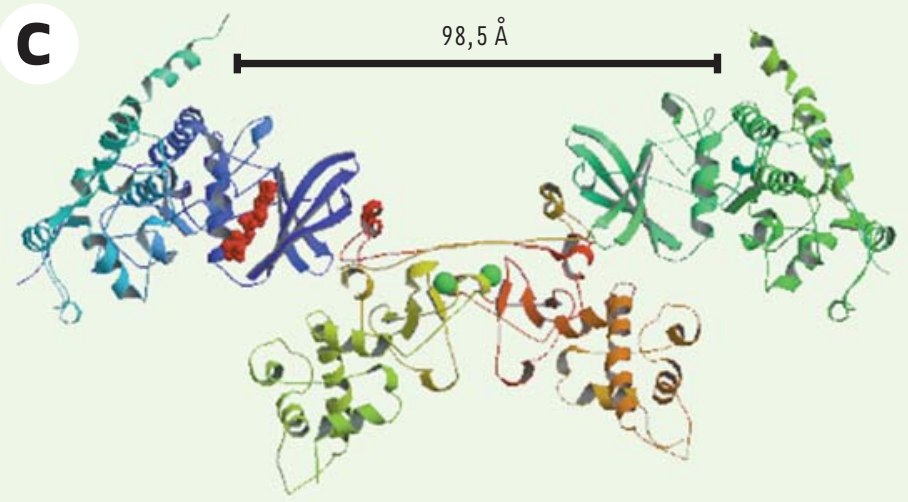

Figure 1. La protéine kinase CK2 à l'échelle atomique. La structure de la sous-unité catalytique (A) révèle la conformation spontanément ouverte de la poche catalytique située entre les deux lobes de la protéine kinase, et rend compte de son activité « constitutive » (adapté de [2]). La structure de la sous-unité régulatrice (B) révèle l'existence d'un domaine extrêmement acide et d'un motif en doigt de zinc responsable de la dimérisation de la protéine. Le dimère ainsi formé présente une large surface accessible pour des interactions avec ses nombreux partenaires. L'extrémité carboxy-terminale de la protéine n'est pas visible sur cette structure (adapté de [3]). La structure de l'holoenzyme (c) montre que les deux sous-unités catalytiques n'interagissent pas entre elles. Une région discrète de nature hydrophobe, située au niveau des feuillets $\beta$ du lobe supérieur de la sous-unité catalytique, interagit avec le domaine carboxy-terminal des deux sous-unités régulatrices. Le caractère flexible de l'interface sous-unité $\alpha$ /sous-unité $\beta$ suggère que l'holoenzyme est un hétérocomplexe transitoire dont la formation est probablement contrôlée dans la cellule (adapté de [6]). 
tions hydrophobes au sein d'un motif original en doigt de zinc [3]. La forte densité en acides aminés conservés exposés à la surface de ce dimère suggère l'existence de domaines d'interaction pour les nombreux partenaires de cette protéine (Figure 3 ). L'importance de CK2 $\beta$ est illustrée par le fait qu'elle exerce des fonctions indépendantes de CK2 $\alpha$ : à titre d'exemple, elle peut régler l'activité de plusieurs protéine kinases impliquées dans des voies de signalisation, telles que c-Mos ou A-Raf $[4,5]$.

La structure tridimensionnelle de l'holoenzyme (Figure 1C) révèle l'architecture d'un complexe en forme de papillon dans lequel le dimère de CK2 $\beta$ sert de plateforme d'assemblage pour deux sous-unités catalytiques [6]. La caractéristique la plus inattendue de cette structure est le caractère flexible des contacts entre les deux sous-unités $\alpha$ et $\beta$ de la CK2, ce qui permet d'envisager l'existence, dans les cellules, d'un équilibre entre des formes associées et non associées des deux protéines. Bien que spéculative, cette notion suggère que l'holoenzyme est un hétérocomplexe transitoire qui peut se former ou se dissocier in vivo pour assurer des fonctions spécifiques.

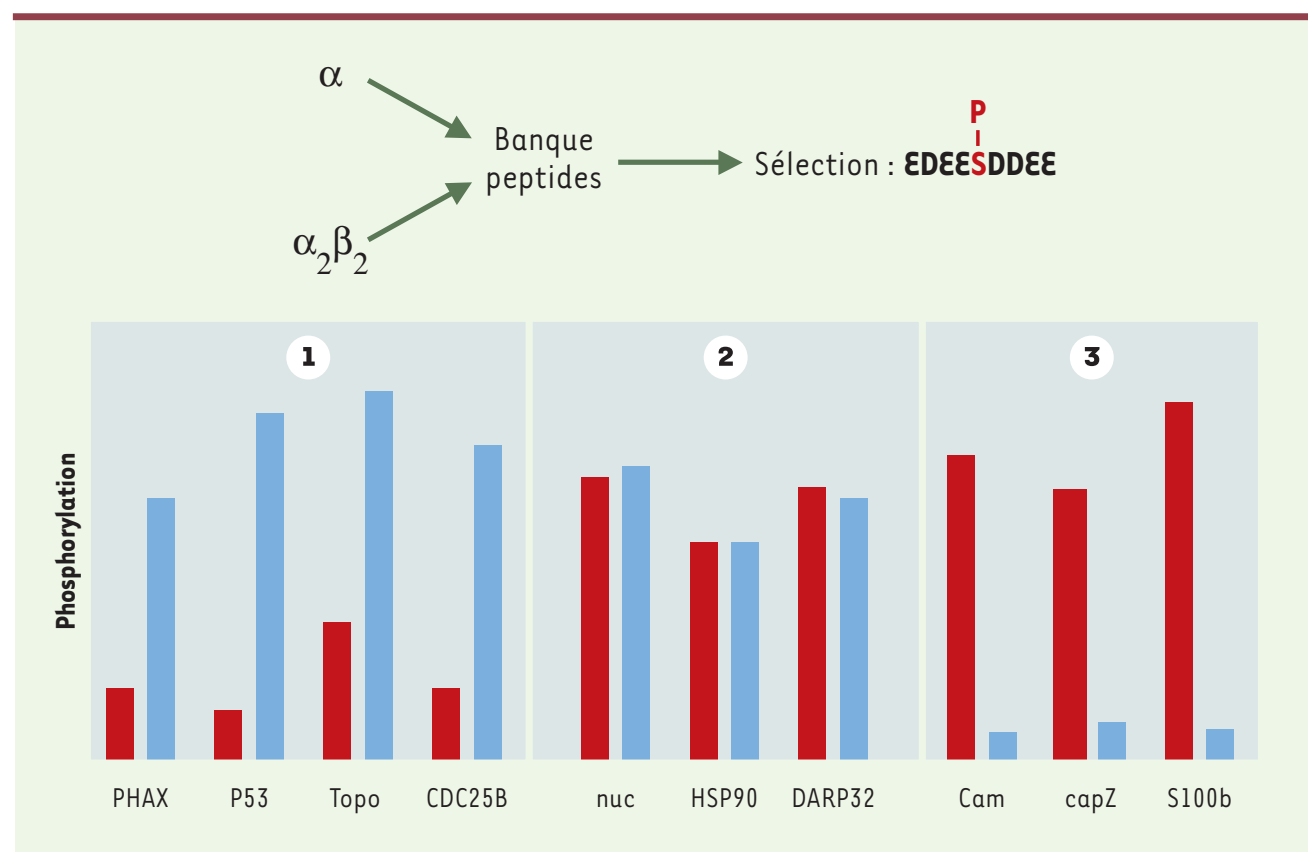

Figure 2. Contrôle de l'activité de la protéine kinase CK2 par sa sous-unité régulatrice. À partir d'une banque contenant $10^{9}$ peptides orientés, les deux formes moléculaires de CK2 ( $\alpha$ et $\alpha 2 \beta 2$ ) phosphorylent de façon optimale la même séquence EDEદSDDEદ [40]. À l'opposé, l'absence (rectangles rouges) ou la présence (rectangles bleus) de la sous-unité $\beta$ règle l'activité de la kinase en contrôlant la reconnaissance de ses substrats protéiques qui peuvent être classés en trois familles. PHAX: protéine d'export des ARN; p53: suppresseur de tumeur; Topo: topo-isomérase II; CDC25B: protéine phosphatase; nuc: nucléoline; HSP90: protéine de choc thermique; DARP32: phosphoprotéine associée aux récepteurs de la dopamine; Cam : calmoduline; capZ: protéine de liaison de l'actine; S100b: protéine de liaison du $\mathrm{Ca}^{2+}$.

\section{Quand localisation rime avec régulation}

La régulation cellulaire de la localisation dynamique («translocation ») des protéines de signalisation est un aspect fondamental de la plupart des voies de transduction. Des études d'immunolocalisation des sous-unités de la CK2 ont montré qu'elles sont majoritairement nucléaires dans les cellules en prolifération. Cependant, dans certaines cellules différenciées (neurones, hépatocytes), une localisation cytoplasmique a été décrite. Par ailleurs, différents compartiments cellulaires (membrane plasmique, cytoplasme, noyau) contiennent des protéines cibles de CK2 [7]. II en découle que les mécanismes contrôlant la localisation de CK2 dans la cellule constituent vraisemblablement un volet important de sa régulation. II a été observé que les sous-unités de CK2 possèdent des séquences de nucléarisation (NLS) qui leur permettent d'être importées, indépendamment l'une de l'autre, dans le noyau cellulaire [8]. Curieusement, bien que toutes les deux soient très mobiles dans ce compartiment, seule la sous-unité catalytique est capable d'être exportée dans le cytoplasme. Enfin, l'association des deux sous-unités - qui peut survenir dans le cytoplasme à la suite de leur néosynthèse conduit à la rétention de l'holoenzyme ainsi formée dans ce compartiment [9]. II ressort de ces différentes études que la localisation, mais également l'interaction des deux sous-unités de CK2 dans la cellule, sont des phénomènes extrêmement dynamiques, dont l'importance fonctionnelle commence à être appréciée: elles seraient déterminantes pour la reconnaissance des substrats de la kinase. Ces paramètres suggèrent également l'existence d'un mécanisme de régulation complexe insoupçonné jusqu'à présent.

\section{CK2 et viabilité cellulaire}

Des études génétiques dans la levure Saccharomyces cerevisiae ont démontré le rôle déterminant de la CK2 dans la viabilité cellulaire. L'in- 
validation des deux gènes codants pour les sous-unités catalytiques est létale. Toutefois, en raison de l'extrême conservation de ces protéines, la viabilité des levures peut être restaurée par l'expression de la protéine de drosophile [10]. Chez la souris, l'extrême homologie fonctionnelle des deux isoformes CK2 $\alpha$ et CK2 $\alpha$ ' et leur présence dans tous les tissus expliquent que les animaux dont le seul gène $C K 2 \alpha^{\prime}$ a été invalidé sont viables. Cependant, CK2 $\alpha$ ' est l'isoforme unique exprimée lors des derniers stades de la spermatogenèse, et l'invalidation du gène pour lequel elle code se traduit donc par une infertilité des mâles due à une altération de la formation antérieure de la tête des spermatides, mimant ainsi la globozoospermie humaine [11].

Les fonctions cellulaires de CK2 $\beta$ ont également été étudiées dans les levures. L'invalidation des gènes CKBI et CKB2 chez S. cerevisiae n'a aucun effet sur les levures cultivées sur milieu normal, mais se traduit par une sensibilité aux cations $\mathrm{Na}^{+}$et $\mathrm{Li}^{+}$[12]. L'absence de la protéine CK2 $\beta$ chez S. pombe entraîne des anomalies de forme de la cellule [13]. Dans les deux cas, il faut noter que l'absence de CK2 $\beta$ ne compromet pas la viabilité cellulaire. À l'opposé, des observations récentes, réalisées dans notre groupe et en collaboration avec le groupe danois de B. Boldyreff, à Odense, montrent que l'invalidation chez la souris du gène unique codant chez les mammifères pour CK2 $\beta$ est létale à un stade précoce du développement embryonnaire, et compromet le développement in vitro de la masse des cellules souches (ES). Ces observations suggèrent que, chez les mammifères, CK2 $\beta$ est indispensable à la viabilité cellulaire [14].

\section{Promotion de la survie cellulaire: une nouvelle fonction de CK2}

Un lien fonctionnel entre CK2 et le cycle de division cellulaire a été établi dans la levure. L'utilisation chez $S$. cerevisiae d'allèles conditionnels thermosensibles pour CK2 $\alpha / \alpha$ ' révèle que CK2 est impliquée dans la progression du cycle cellulaire, au niveau de la phase $\mathrm{Gl}$ et de la transition $G 2 \rightarrow M[15]$. Dans les cellules de mammifères, des expériences utilisant la micro-injection d'anticorps neutralisants ou d'oligonucléotides antisens (anti- $\alpha$ et/ou anti- $\beta$ ), ou l'expression de formes inactives des sous-unités catalytiques de la CK2 ont permis d'établir que les sous-unités de la kinase sont là aussi impliquées dans le contrôle de la division cellulaire [1620]. L'intervention de la CK2 dans la promotion ou l'exécution de certaines phases du cycle cellulaire est à mettre en parallèle avec l'observation selon laquelle la plupart des cancers possèdent une activité élevée de la CK2 [21]. À titre d'exemples, les deux sous-unités de la kinase sont surexprimées dans les tumeurs du sein et dans des tumeurs mammaires induites par des carcinogènes chez le rat [22]. Le potentiel oncogénique de la CK2 a été révélé par une surexpression ciblée et modérée de la CK2 dans des modèles de souris transgéniques [23, $24]$. Cependant, il faut noter qu'une surexpression des sous-unités $\alpha$ et $\beta$ de la CK2 dans des fibroblastes ou dans des cellules d'ostéosarcomes ne s'accompagne d'aucun changement phénotypique cellulaire apparent $[20,25]$. Dans ce contexte, il est plausible qu'un dérèglement de cette enzyme puisse conduire à une phosphorylation aberrante de protéines impliquées non pas dans la prolifération incontrôlée, mais plutôt dans la survie des cellules cancéreuses.

Plusieurs observations récentes révèlent des connexions biochimiques et fonctionnelles entre la CK2 et plusieurs acteurs moléculaires assurant la survie des cellules cancéreuses en les protégeant, notamment, du processus apoptotique (Figure 4).

Au cours d'une réponse inflammatoire, le facteur de transcription NF-KB déclenche l'activation de gènes anti-apoptotiques. NF-KB est normalement maintenu captif sous une forme inactive dans le cytoplasme par son association avec la protéine inhibitrice $\mid \kappa B$. CK2 fait

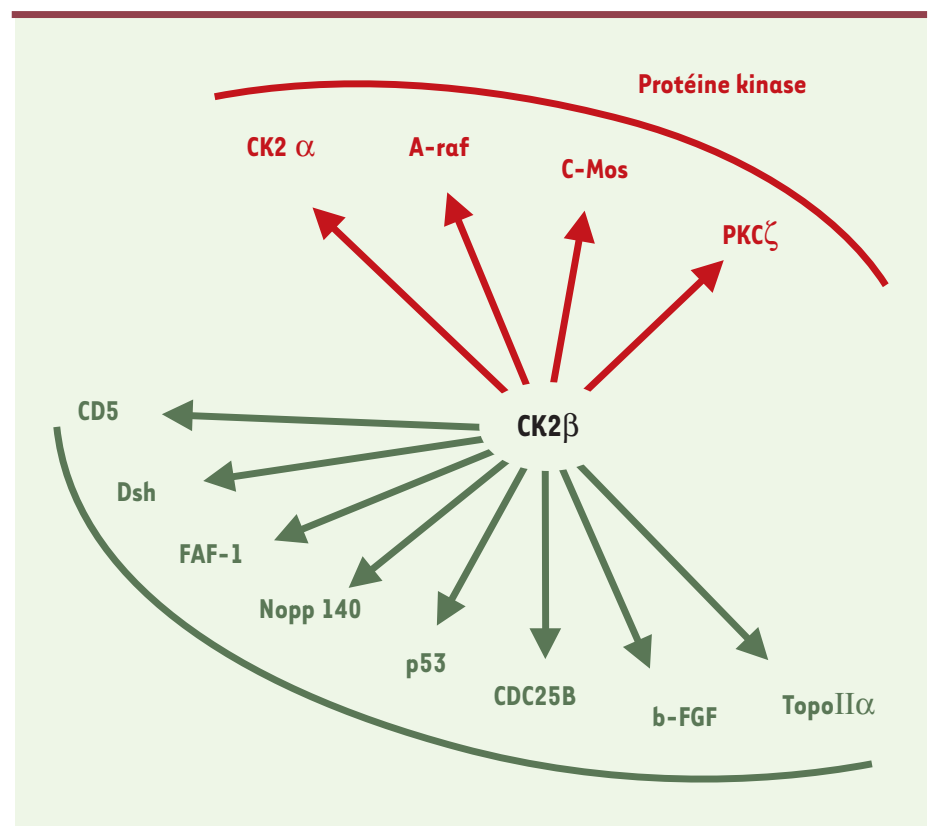

Figure 3. Diversité des partenaires de CK2 $\beta$. De nombreux partenaires protéiques de la CK2 $\beta$ ont été identifiés par des techniques de co-immunoprécipitation ou de criblage en double hybride dans la levure. CK2 $\beta$ interagit par son extrémité carboxy-terminale avec CK2 $\alpha$ [41], mais également avec d'autres protéine kinases

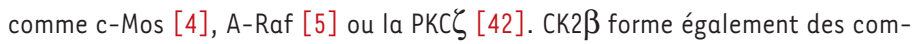
plexes stables avec des protéines membranaires ou cytoplasmiques telles que CD5 [43], Dsh [44], FAF-1 [41], ou des protéines nucléaires comme Nopp140 [45], P53 [46], la topoisomérase II $\alpha$ [47], b-FGF [48] ou CDC25B [49]. 
partie des kinases qui règlent la dégradation de IKB [26] et stimulent l'activité transcriptionnelle de NF-KB

$[27,28]$. Le fait que le promoteur du gène de CK2 $\alpha$ soit régulé par NF-KB permet d'envisager l'existence d'une boucle de rétrocontrôle positif conduisant à une surexpression concertée de CK2 $\alpha$ et de NF-KB dans certains cancers [29].

La voie de signalisation dépendante du ligand Wnt-l est réactivée dans de nombreux cancers, où elle contribue à l'augmentation de la survie cellulaire en bloquant

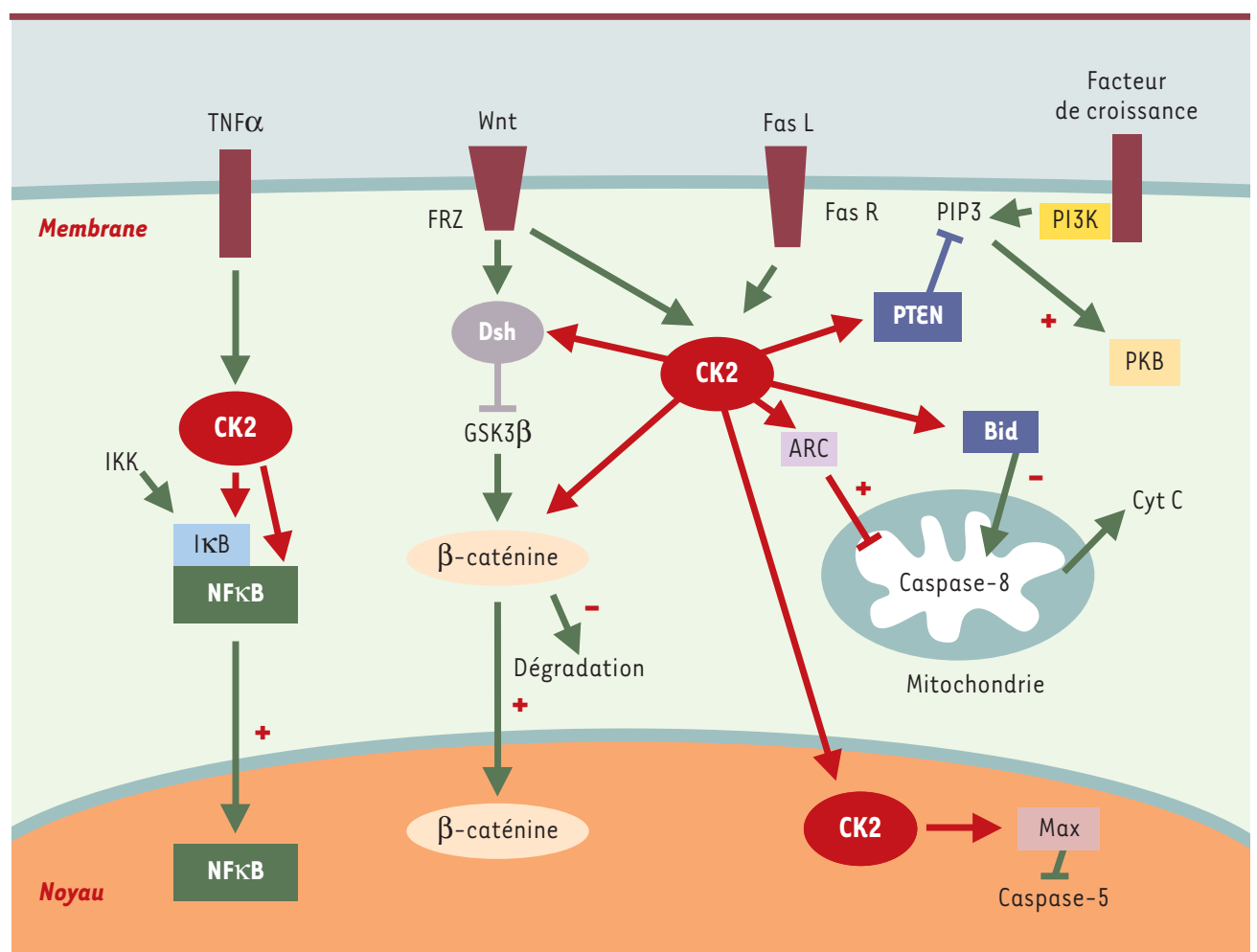

Figure 4. Implication de la protéine kinase CK2 dans la survie cellulaire. Lors d'une réponse inflammatoire, la protéine inhibitrice $\mid \kappa B$, qui maintient normalement dans le cytoplasme le facteur de transcription NF- $K B$ sous une forme inactive, est dégradée. La CK2 fait partie des kinases qui règlent la dégradation de IKB et stimulent l'activité transcriptionnelle de NF-KB. La voie de signalisation dépendante du ligand Wnt-l contribue à l'augmentation de la survie cellulaire en bloquant l'apoptose [30]. La CK2, présente dans un complexe contenant, entre autres, la protéine Dsh et la $\beta$-caténine, participe à la transduction du signal Wnt-1. La phosphorylation de la $\beta$-caténine par CK2 stimule sa translocation nucléaire et règle l'expression de gènes dont les produits protéiques sont impliqués dans la survie celIulaire [31]. Lors de l'activation du récepteur Fas, la protéine pro-apoptotique Bid doit être clivée par la caspase-8 pour permettre la libération du cytochrome C [36]. La phosphorylation de Bid par la CK2 sur un site proche du site de coupure de la caspase-8 la rend résistante à cette protéolyse activatrice [37]. La phosphorylation par CK2 de la protéine ARC, protéine inhibitrice de la caspase-8, cible cette protéine dans la mitochondrie et permet ainsi son interaction avec la caspase [38]. De façon similaire, l'activation du récepteur Fas déclenche la protéolyse du facteur de transcription Max par la caspase 5. Là encore, la phosphorylation de Max par la CK2 rend ce facteur de transcription résistant à cette protéolyse [39]. La PI3K est nécessaire et suffisante pour protéger les cellules de l'apoptose. Le produit du gène suppresseur de tumeur PTEN antagonise l'action anti-apoptotique de la PI3K [33]. La phosphorylation de PTEN par CK2 entraîne une inhibition de son activité phosphatase [34]. TNF $\alpha$ : tumor necrosis factor $\alpha$; FRZ: récepteur frizzled; Dsh: dishevelled; GSK3 $\beta$ : glycogen synthase kinase $3 \beta$; Fas L: Fas ligand; Fas R: récépteur de Fas L; PI3K: phosphatidylinositol 3-kinase; Cyt C: cytochrome C; PKB: protéine kinase B, IKK: IKB-kinase. l'apoptose [30]. Un complexe multiprotéique intracellulaire contenant, entre autres éléments, la protéine Dishvelled (Dsh) et la $\beta$-caténine participe à la transduction du signal. En présence du ligand Wnt-1, la CK2, également présente au sein de ce complexe, phosphoryle la $\beta$-caténine et stimule sa translocation nucléaire [31]. La $\beta$ caténine peut alors, à En permettant l'activation de la protéine kinase Akt/PKB, la phosphatidynte pour protéger les cellules de l'apoptose [32]. Le produit du gène suppresseur de tumeur PTEN possède une activité phosphatase qui antagonise l'action anti-apoptotique de la PI3K [33]. La protéine PTEN est phosphorylée par CK2 sur son extrémité régulatrice carboxy-terminale. Cette phosphorylation règle négativement l'activité de la phosphatase en bloquant son intégration dans un complexe macromoléculaire nécessaire pour son adressage à la membrane plasmique [34]. En neutralisant PTEN, la CK2 potentialise l'action essentielle de la PI3K et de la PKB (protéine kinase B) pour assurer la survie cellulaire.

D'un point de vue expérimental, une forte induction de l'apoptose est observée dans des cellules cancéreuses dans lesquelles l'expression de l'une ou l'autre des deux sous-unités de la CK2 a été perturbée par des oligonucléotides antisens [35]. Parallèlement, l'apoptose chimio-induite de cellules cancéreuses de la prostate est bloquée par une surexpression de CK2 $\alpha$, ce qui suggère que la CK2 participe au blocage massif de la machinerie apoptotique, 
souvent observé lors des protocoles de chimiothérapie. Une interaction fonctionnelle entre CK2 et les mécanismes impliqués dans l'apoptose a été récemment mise en évidence. La protéine pro-apoptotique Bid, qui appartient à la famille des protéines $\mathrm{Bcl}-2$, joue un rôle crucial dans l'apoptose induite par le récepteur Fas. Bid doit être clivée par la caspase-8, la translocation dans la mitochondrie du fragment actif ainsi produit permettant la libération du cytochrome $C$ et l'activation en cascade de diverses caspases [36]. Or, la phosphorylation de Bid par la CK2 sur un site proche du site de coupure de la caspase8 rend la protéine résistante à cette protéolyse activatrice [37]. Cette étude a permis d'établir pour la première fois que la surexpression de la sous-unité catalytique $\alpha$ de la protéine CK2 protège les cellules de l'apoptose induite par le récepteur Fas. Par ailleurs, la caspase-8 possède un inhibiteur protéique cytoplasmique, la protéine ARC. Sa phosphorylation par la CK2 cible cette protéine inhibitrice dans la mitochondrie et permet son interaction avec la caspase-8 [38]. La CK2, en régulant négativement l'activité de la caspase-8, participe donc directement aux mécanismes contrôlant la survie cellulaire.

Les protéines Myc/Max/Mad sont des régulateurs transcriptionnels jouant un rôle crucial dans le contrôle de la prolifération, de la différenciation et de l'apoptose cellulaires. Il a été observé que Max est clivé par les caspases- 5 et 7 lors de l'apoptose déclenchée par l'activation de Fas, cette protéolyse de Max modifiant ses propriétés de liaison à I'ADN. De façon similaire à celle observée pour Bid, la phosphorylation de Max par CK2 rend ce facteur de transcription résistant à la protéolyse par la caspase-5 [39].

Il faut noter, dans ce contexte, qu'il existe une forte similitude entre la séquence de reconnaissance de protéolyse par les caspases et le motif consensus pour la phosphorylation par la CK2.

II existe donc un lien fonctionnel entre la CK2 et la machinerie propageant des signaux apoptotiques. Dans ce contexte, la surexpression de la CK2 observée dans toutes les tumeurs pourrait refléter sa participation, à côté d'autres « kinases de survie », à la neutralisation des programmes d'apoptose nécessaire à la propagation des cellules cancéreuses.

\section{Conclusions}

Bien qu'étant connue depuis plusieurs décennies, la CK2 est restée pendant longtemps en marge des investigations de la biologie cellulaire. Son absence apparente de régulation, ses propriétés enzymatiques et le nombre considérable de ses substrats potentiels ont contribué à son image de protéine kinase «à part ». Cependant, les derniers développements obtenus sur sa structure révèlent une architecture moléculaire compatible avec sa dynamique intracellulaire. Le rôle crucial de ses sousunités pour la viabilité cellulaire dans la levure, mais également au cours du développement embryonnaire de la souris, a été clairement mis en évidence par des approches génétiques. L'observation d'une surexpression des sous-unités de la kinase dans tous les cancers peut pour l'instant n'être qu'une coïncidence; il faut toutefois noter que le caractère oncogénique de la CK2 a été établi dans plusieurs systèmes expérimentaux. Ces caractéristiques, couplées aux observations suggérant que cette enzyme participe aux signaux de survie dans les cellules, renforcent la notion de son intégration dans les processus complexes de cancérogenèse.

La CK2, protéine kinase « à part », a récemment bénéficié d'une approche multidisciplinaire et a ainsi acquis une « maturité » lui permettant, désormais, de s'intégrer dans des voies de signalisation essentielles pour la cellule. On peut s'attendre à ce que les connaissances accumulées sur cette protéine kinase au cours des dernières années conduisent à des développements futurs fructueux. $\diamond$

\section{REMERCIEMENTS}

Nous remercions vivement Odile Filhol pour la relecture $d u$ manuscrit. Les travaux du groupe Structure et fonctions de la CK2 de l'EMI 0104 bénéficient du soutien financier de l'Inserm, du CEA, du Cnrs (contrat MCT 01-05), de la Ligue Nationale pour la Recherche contre le Cancer et de l'Association pour la Recherche contre le Cancer (contrat ARECA).

\section{SUMMARY}

Protein kinase CK2:

an enzyme that likes to be different

Protein kinase CK2 (formerly known as casein kinase 2) was among the first protein kinases to be identified and characterized. Surprisingly, in spite of intense efforts, the regulation and cellular functions of CK2 remain obscure. However, recent data on its molecular structure, its signal-mediated intracellular dynamic localization and its unexpected function in cell survival have raised new interest in this enzyme. These studies reveal unique features of CK2 and highlight its importance in the transduction of survival signals. $\diamond$ 


\section{RÉFÉRENCES}

1. Litchfield DW. Protein kinase CK2: structure, regulation and role in cellular decisions of life and death. Biochem J 2003; 369: 1-15.

2. Niefind K, Guerra B, Pinna $L A$, Issinger $O G$, Schomburg D. Crystal structure of the catalytic subunit of protein kinase CK2 from Zea mays at 2.1 A resolution. EMBO J 1998; 17: 2451-62.

3. Chantalat L, Leroy D, Filhol 0 , et al. Crystal structure of the human protein kinase CK2 regulatory subunit reveals its zinc fingermediated dimerization. EMBO J 1999; 18: 2930-40.

4. Chen M, Cooper JA. The $\beta$ subunit of CKII negatively regulates Xenopus oocyte maturation. Proc Natl Acad Sci USA 1997; 94: 9136-40.

5. Boldyreff $B$, Issinger OG. ARaf kinase is a new interacting partner of protein kinase CK2 $\beta$ subunit. FEBS Lett 1997; 403: 197-9.

6. Niefind K, Guerra B, Ermakowa I, Issinger OG. Crystal structure of human protein kinase CK2: insights into basic properties of the CK2 holoenzyme. EMBO J 2001; 20: 5320-31.

7. Pinna LA. Casein kinase 2 : an eminence grise in cellular regulation? Biochim Biophys Acta 1990; 1054: 267-84.

8. Martel V, Filhol 0, Nueda A, Gerber D, Benitez MJ, Cochet C. Visualization and molecular analysis of nuclear import of protein kinase CK2 subunits in living cells. Mol Cell Biochem 2001; 227: 81-90.

9. Filhol 0, Nueda A, Martel V, et al. Live cell fluorescence imaging reveals the dynamics of protein kinase CK2 individual subunits. Mol Cell Biol 2003 (sous presse)

10. Padmanabha R, Chen-Wu JL, Hanna DE, Glover CV. Isolation, sequencing, and disruption of the yeast CKA2 gene: casein kinase II is essential for viability in
Saccharomyces cerevisiae. Mol Cell Biol 1990; 10: 4089-99.

11. Xu X, Toselli PA, Russell LD, Seldin DC. Globozoospermia in mice lacking the casein kinase II $\alpha$ ' catalytic subunit. Nat Genet 1999; 23: 118-21

12. Bidwai AP, Reed JC, Glover CV. Cloning and disruption of $C K B 1$, the gene encoding the $38-k D a \beta$ subunit of Saccharomyces cerevisiae casein kinase II (CKII). Deletion of CKII regulatory subunits elicits a saltsensitive phenotype. J Biol Chem 1995; 270: 10395-404.

13. Roussou I, Draetta G. The Schizosaccharomyces pombe casein kinase $\| \alpha$ and $\beta$ subunits: evolutionary conservation and positive role of the $b$ subunit. Mol Cell Biol 1994; 14: 576-86.

14. Buchou T, Vernet M, Blond 0 , et al. Disruption of the regulatory subunit of protein kinase CK2 in mice leads to a cell-autonomous defect and early embryonic lethality. Mol Cell Biol; 2003 23: 908-15.

15. Hanna DE, Rethinaswamy $A$ Glover CV. Casein kinase II is required for cell cycle progression during $\mathrm{Gl}$ and G2/M in Saccharomyces cerevisiae. J Biol Chem 1995; 270: 25905-14.

16. Lorenz P, Pepperkok R, Ansorge W, Pyerin W. Cell biological studies with monoclonal and polyclonal antibodies against human casein kinase II subunit $\beta$ demonstrate participation of the kinase in mitogenic signaling. J Biol Chem 1993; 268: 2733-9.

17. Pepperkok R, Lorenz $P$, Jakobi R, Ansorge W, Pyerin $W$. Cell growth stimulation by EGF: inhibition through antisense-

oligodeoxynucleotides demonstrates important role of casein kinase II. Exp Cell Res 1991; 197: 245-53.

18. Li D, Dobrowolska G, Aicher LD, et al. Expression of the casein kinase 2 subunits in
Chinese hamster ovary and $3 T 3 \mathrm{Ll}$ cells provides information on the role of the enzyme in cell proliferation and the cell cycle. J Biol Chem 1999; 274: 32988-96.

19. Vilk G, Saulnier RB, St Pierre $R$, Litchfield DW. Inducible expression of protein kinase CK2 in mammalian cells. Evidence for functional specialization of CK2 isoforms. J Biol Chem 1999; 274: 14406-14.

20. Lebrin F, Chambaz EM, Bianchini L. A role for protein kinase CK2 in cell proliferation: evidence using a kinase-inactive mutant of CK2 catalytic subunit $\alpha$. Oncogene 2001; 20: 2010-22.

21. Tawfic $S$, $Y u S$, Wang $H$ Faust R, Davis A, Ahmed K. Protein kinase CK2 signal in neoplasia. Histol Histopathol 2001; 16: 573-82.

22. Landesman-Bollag $\varepsilon$, Romieu-Mourez R, Song DH, Sonenshein GE, Cardiff RD, Seldin DC. Protein kinase CK2 in mammary gland tumorigenesis. Oncogene 2001; 20: 3247-57.

23. Seldin DC, Leder P. Casein kinase II $\alpha$ transgeneinduced murine lymphoma: relation to theileriosis in cattle. Science 1995; 267 : 894-7.

24. Landesman-Bollag $\varepsilon$, Channavajhala PL, Cardiff RD, Seldin DC. p53 deficiency and misexpression of protein kinase CK $2 \alpha$ collaborate in the development of thymic lymphomas in mice. Oncogene 1998; 16 : 2965-74.

25. Vilk G, Derksen DR, Litchfield DW. Inducible expression of the regulatory protein kinase CK2 $\beta$ subunit: incorporation into complexes with catalytic CK2 subunits and reexamination of the effects of CK2 $\beta$ on cell proliferation. J Cell Biochem 2001; 84: 84-99.

26. Romieu-Mourez R, Landesman-Bollag $\varepsilon$, Seldin
DC, Traish AM, Mercurio F, Sonenshein GE. Roles of IKK kinases and protein kinase CK2 in activation of nuclear factor- $K B$ in breast cancer. Cancer Res 2001; 61: 3810-8.

27. Wang D, Westerheide SD, Hanson JL, Baldwin AS, Jr. Tumor necrosis factor $\alpha$ induced phosphorylation of RelA/p65 on Ser529 is controlled by casein kinase II. J Biol Chem 2000; 275: 32592-7.

28. Bird TA, Schooley K, Dower SK, Hagen H, Virca GD.

Activation of nuclear transcription factor NF- KB by interleukin-l is accompanied by casein kinase II-mediated phosphorylation of the $\mathrm{p} 65$ subunit. J Biol Chem 1997; 272: 32606-12.

29. Krehan A, Ansuini H, Bocher 0 , Grein $S$, Wirkner U, Pyerin $W$. Transcription factors ets $\mathrm{N}, \mathrm{NF}-\mathrm{K} \mathrm{B}$, and $\mathrm{Spl}$ are major determinants of the promoter activity of the human protein kinase CK2 $\alpha$ gene. J Biol Chem 2000; 275: 18327-36.

30. Chen S, Guttridge DC, You Z et al. Wnt-1 signaling inhibits apoptosis by activating $\beta$-catenin $/ T$ cell factor-mediated transcription. J Cell Biol 2001; 152: 87-96.

31. Song DH, Sussman DJ, Seldin DC. Endogenous protein kinase CK2 participates in Wnt signaling in mammary epithelial cells. J Biol Chem 2000; 275: 23790-7.

32. Stambolic V, Mak TW, Woodgett JR. Modulation of cellular apoptotic potential: contributions to oncogenesis. Oncogene 1999; 18: 6094-103.

33. Yamada KM, Araki M. Tumor suppressor PTEN: modulator of cell signaling, growth, migration and apoptosis. J Cell Sci 2001; 114 : 2375-82.

34. Torres J, Pulido R. The tumor suppressor PTEN is phosphorylated by the protein kinase CK2 at its C terminus. Implications for PTEN stability to 
proteasome-mediated degradation. J Biol Chem 2001; 276: 993-8.

35. Wang H, Davis A, Yu S, Ahmed K. Response of cancer cells to molecular interruption of the CK2 signal. Mol Cell Biochem 2001; 227: 167-74.

36. Li H, Zhu H, Xu CJ, Yuan J. Cleavage of BID by caspase 8 mediates the mitochondrial damage in the Fas pathway of apoptosis. Cell 1998; 94 : 491-501.

37. Desagher $S, 0$ sen-Sand A, Montessuit S, et al. Phosphorylation of Bid by casein kinases I and II regulates its cleavage by caspase 8. Mol Cell 2001; 8: 601-11.

38. Li PF, Li J, Muller \&C, Otto A, Dietz $R$, von Harsdorf $R$. Phosphorylation by protein kinase CK2: a signaling switch for the caspaseinhibiting protein ARC. Mol Cell 2002; 10: 247-58.
39. Krippner-Heidenreich $A$, Talanian RV, Sekul R, et al. Targeting of the transcription factor Max during apoptosis: phosphorylation-regulated cleavage by caspase- 5 at an unusual glutamic acid residue in position $\mathrm{Pl}$. Biochem / 2001; 358: 705-15.

40. Songyang Z, Lu KP, Kwon YT, et al. A structural basis for substrate specificities of protein Ser/Thr kinases: primary sequence preference of casein kinases I and II, NIMA, phosphorylase kinase, calmodulin-dependent kinase II, CDK5, and Erkl. Mol Cell Biol 1996; 16: 6486-93.

41. Kusk M, Ahmed R, Thomsen $B$, Bendixen $C$, Issinger $O G$, Boldyreff $B$. Interactions of protein kinase CK2 $\beta$ subunit within the holoenzyme and with other proteins. Mol Cell Biochem 1999; 191: 51-8.

42. Bren GD, Pennington KN,
Paya CV. PKC-zeta associated CK2 participates in the turnover of free $1 \kappa B$ a. J Mol Biol 2000; 297 : 1245-58.

43. Raman C, Kuo A, Deshane J, Litchfield DW, Kimberly RP. Regulation of casein kinase 2 by direct interaction with cell surface receptor CD5. J Biol Chem 1998; 273: 19183-9.

44. Willert K, Brink M, Wodarz A Varmus $H$, Nusse R. Casein kinase 2 associates with and phosphorylates dishevelled. EMBO J 1997 ; 16: 3089-96.

45. Li D, Meier UT, Dobrowolska $G$, Krebs \&G. Specific interaction between casein kinase 2 and the nucleolar protein Nopp 140. J Biol Chem 1997; 272: 3773-9.

46. Filhol 0, Baudier J, Delphin C, Loue-Mackenbach P, Chambaz EM, Cochet $C$. Casein kinase II and the tumor suppressor protein P53 associate in a molecular complex that is negatively regulated upon p53 phosphorylation. J Biol Chem 1992; 267: 20577-83.

47. Bojanowski K, Filhol 0 , Cochet C, Chambaz EM, Larsen AK. DNA topoisomerase II and casein kinase II associate in a molecular complex that is catalytically active. J Biol Chem 1993; 268: 22920-6.

48. Bonnet $\mathrm{H}$, Filhol 0 , Truchet I, et al. Fibroblast growth factor-2 binds to the regulatory $\beta$ subunit of CK2 and directly stimulates CK2 activity toward nucleolin. J Biol Chem 1996; 271 : 24781-7.

49. Theis-Febvre N, Filhol 0, Froment $\mathrm{C}$, et al. Protein kinase CK2 regulates CDC25B phosphatase activity. Oncogene 2003; 22: 220-32.

\section{TIRÉS À PART}

T. Buchou

\section{PUB Immunologie Flammarion Film chez Corlet}

